\section{Endoclip-Assisted Closure of Infected Gastrocutaneous Fistulas: Report of Two Cases}

A rare complication after the removal of a percutaneous endoscopic gastrostomy (PEG) is the presence of a refractory gastrocutaneous fistula, the overall incidence of which has not been reported to date. Usually, after a PEG is removed, the fistula closes within $48-72 \mathrm{~h}$. A major reason for gastrocutaneous fistulas remaining open is the presence of fibrosis.

Two patients who underwent PEG tube removal developed infected gastrocutaneous fistulas, for which they were admitted to hospital and treated with conservative measures (a clear liquid diet, parenteral nutrition, and intravenous administration of a histamine blocker for 5

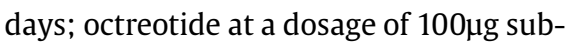
cutaneously every $8 \mathrm{~h}$ for $72 \mathrm{~h}$; and intravenous levofloxacin to treat the cellulitis surrounding the fistulas). Despite these measures, the fistulas did not close. The patients were not candidates for surgery.

Endoscopy was carried out with topical anesthetic and conscious sedation. During endoscopy, the gastric side of the fistula was identified (Figure $\mathbf{1}$ ). The cutaneous side of the fistula was cleaned and $1 \%$ lignocaine (Xylocaine) was injected into the fistula. Under endoscopic guidance, the BICAP electrocautery probe (Circon Corporation, Santa Barbara, California, USA) was then inserted through the abdominal side of the fistula into the stomach and the fistula tract was cauterized (with a setting of $20 \mathrm{~W}$ ) by passing the probe twice through the gastrocutaneous fistula for approximately $3 \mathrm{~s}$. Next, mechanical closure of the gastric side of the fistula was achieved using two stainless-steel Endoclips (Figure 2). Then, $0.5 \mathrm{ml}$ of a topical skin adhesive (2-octylcyanoacrylate; Dermabond, Ethicon, Inc., Piscataway, New Jersey, USA) was injected through the abdominal side into the fistula tract. Two weeks after the patients had been discharged from the hospital, examination showed that the fistulas in both patients had healed.

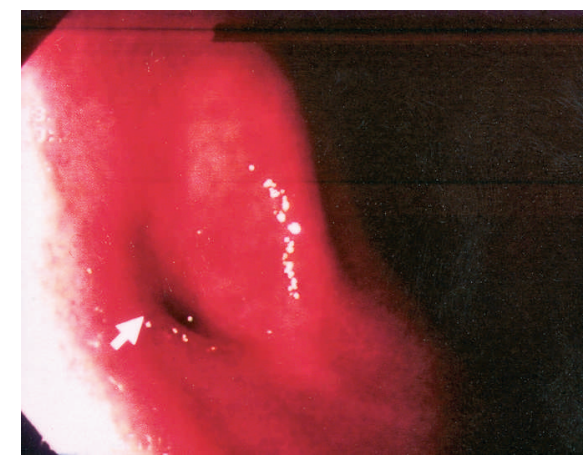

Corresponding Author

\section{J. J. Alberti-Flor, M.D., F.A.C.P}

2601 S.W. 37th Avenue

Suite 506

Miami, FL 33133

USA

Fax: $\quad+1-305-444-7342$

E-mail: AlbertiFl@aol.com
Figure 1 Endoscopic view of the gastric side of a gastrocutaneous fistula (arrow).

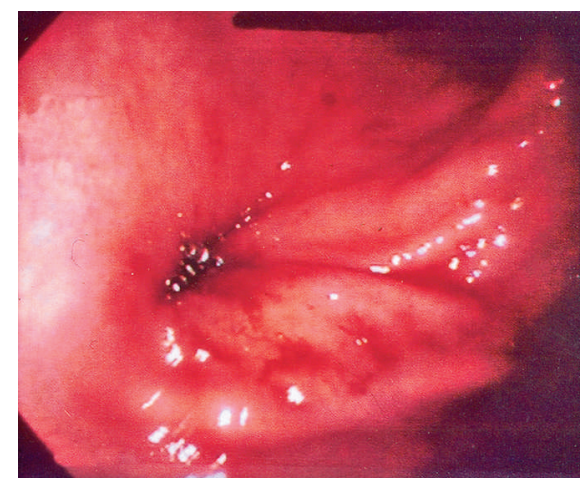

Figure 2 Endoscopic view showing the two Endoclips closing the gastric side of the gastrocutaneous fistula.

This innovative technique for closing gastrocutaneous fistulas using electrocautery (to destroy fibrous tissue), Endoclips (to achieve mechanical closure on the gastric side of the fistula, improving the adherence of the fistula walls and preventing the flow of gastric secretions), and topical skin adhesive (to seal the fistula opening from the abdominal side) may prove useful in other patients suffering from refractory gastrocutaneous fistulas.

\section{E. Alberti ${ }^{1}$, J. J. Alberti-Flor ${ }^{2}$}

${ }^{1}$ Student, Nova Southeastern College of Osteopathic Medicine, Fort Lauderdale, Florida, USA

${ }^{2}$ Division of Gastroenterology, Mercy Hospital, Miami, Florida, USA. 